\title{
МАТЕМАТИЧЕСКАЯ ОБРАБОТКА ЭКСПЕРИМЕНТОВ ПО ФТОРИДНОЙ ПЕРЕРАБОТКЕ НЕФЕЛИНОВ
}

Пушкин А.А., Римкевич В.С.

ИГиП ДВО РАН, Благовещенск, igip@ascnet.ru

\section{Аннотация}

В статье описывается программа, использующая для расчётов по химической кинетике регрессионный и корреляционный анализ. Помимо определения кинетических характеристик гетерофазной реакции производится проверка статистических гипотез об адекватности используемых моделей регрессии при помощи критерия Фишера, о значимости коэффициентов регрессий с использованием t-критерия Стьюдента и проверка практической значимости моделей регрессии с использованием показателя согласованности Стьюдента. Расчёт проведён для реакции фторирования нефелинового концентрата Кольского полуострова гидродифторидом аммония.

\section{Summary}

The program using regression and correlation analysis for chemical kinetics computation is described in paper. The test of statistical hypotheses of adequateness of using regression models with using of Snedecor-Fisher criterion, about the significance of regression coefficients by t-criterion of Student and of practical significance of using regression models with using of Student cooperation index is used besides of kinetic characteristic computation of heterophase reaction. The calculation is performed for reaction of fluorination of nepheline concentrate of Kolskiy peninsula by ammonium hydrodifluoride.

Разработка фторидной и гидрофторидной технологий переработки минерального силикатного и алюмосиликатного сырья в Институте геологии и природопользования ДВО РАН требует проведения предварительного термодинамического расчёта и математической обработки результатов экспериментов $[1,2]$. С этой целью на языке Visual Basic созданы программы Потенциалы и Кинетика, соответственно.

Данная работа посвящена компьютерной обработке экспериментов по фторированию нефелиновых концентратов гидродифторидом аммония, в ходе которых после улетучивания водяного пара и аммиака образовывался твёрдый остаток, состоящий из гексафторидов и фторидов. В экспериментах измерялась убыль массы твёрдого остатка в последовательные моменты времени от 0 до 6.5 часов при четырёх температурах $100,150,175$ и $200^{\circ} \mathrm{C}$. Изменения массы пересчитывались в степени превращения вещества и служили отправной точкой для расчётов.

Результатами обработки экспериментальных данных являются константы скоростей, порядки реакций и энергии активации, а также зоны протекания и механизмы реакций.

Исследуемая реакция фторирования нефелина гетерофазная, т.к. реагенты находились в твёрдых фазах, а продукты - в твёрдых и газообразных. 
Гетерофазные реакции обычно протекают в две стадии: химическое взаимодействие частиц и диффузия реагентов к фронту реакции, а также продуктов реакции от него [3]. Если определяющим является процесс химического взаимодействия между частицами, то реакция протекает в кинетической зоне, если диффузия - то в диффузионной зоне [4].

С целью определения механизма реакции в работе проводилась проверка на лучшую аппроксимацию реального физико-химического процесса четырьмя различными видами физико-химических процессов, соответствующих четырём законам изменения степени превращения вещества: степенному $w_{j}=k \cdot \alpha_{j}^{m}$, Авраами $\alpha_{j}=1-\exp \left(-k \cdot t_{j}^{m}\right)$, экспоненциальному $\alpha_{j}=1-\exp \left(-k \cdot t_{j}\right)$ и Праута-Томпкинса $\alpha_{j}=\frac{\exp \left(-k \cdot t_{j}\right)}{1+\exp \left(-k \cdot t_{j}\right)}$, где $w_{j}-$ скорость реакции, $\alpha_{j}-$ степень превращения вещества в момент времени $t_{j}, k$ - константа скорости. Степенной закон отражает закон действующих масс и описывает столкновения между частицами, он же должен описывать реакцию в кинетической зоне. Уравнение Авраами является топохимическим и описывает химическую реакцию в диффузионной зоне. Экспоненциальное уравнение описывает химические реакции первого порядка [5], и, в этом смысле, является частным случаем уравнения Авраами. Уравнение Праута-Томпкинса описывает цепные реакции с ветвлением $[5,6]$.

Аппроксимация экспериментальных данных испытуемыми температурными зависимостями оценивается по значениям средних погрешностей аппроксимаций. В первом приближении механизм реакции при данной температуре определяется тем законом изменения концентраций, при котором средняя погрешность аппроксимации при данной температуре минимальна. Поскольку погрешности аппроксимаций вычисляются для каждой температуры, постольку механизм реакций для каждой температуры может быть своим. В программе организован автоматический отбор данных (констант скоростей, энергий активации, зон и механизмов реакций) для каждой из исследуемых температур [7].

Методами исследования в работе являются регрессионный и корреляционный анализы. Для каждого из упомянутых выше четырёх процессов строятся уравнения регрессий. Полученные уравнения регрессий нелинейны. Вводя замены переменных, указанные в табл. 1, осуществляем переход к обобщённой линейной модели регрессии:

$$
y_{i j}=b_{1 i} \cdot x_{j}+b_{0 i} \text {, }
$$

где индекс $i$ означает вид опытной функции (1 - степенной закон, 2 - Авраами, 3 - экспоненциальный закона и 4 - уравнение Праута-Томпкинса), индекс $j$ нумерует моменты отсчета времени $t_{j}$ и соответствующие значения степеней превращения вещества $\alpha_{j}$. 
Таблица 1. Замены переменных при переходе от нелинейных моделей к обобщенной линейной модели.

\begin{tabular}{|c|c|c|c|c|}
\hline & Степенной & Авраами & Экспоненциальный & Праута-Томпкинса \\
\hline$x$ & $\ln \alpha$ & $\ln t$ & $-t$ & $-t$ \\
\hline$y$ & $\ln w$ & $\ln (-\ln (1-\alpha))$ & $\ln (1-\alpha)$ & $\frac{\ln \frac{\alpha}{1-\alpha}}{k}$ \\
\hline$b_{1}$ & $m$ & $m$ & $k$ & 0 \\
\hline$b_{0}$ & $\ln k$ & $\ln k$ & 0 & $k$ \\
\hline
\end{tabular}

Далее, по формулам метода наименьших квадратов вычисляем значения угловых коэффициентов и свободных членов. Как видно из пояснений к формуле (1) в случае степенного закона и уравнения Авраами угловые коэффициенты равны соответствующим порядкам реакций, а свободные члены - логарифмам констант скоростей. В случае экспоненциального закона и уравнения Праута-Томпкинса угловые коэффициенты представляют собой константы скоростей, свободные члены отсутствуют.

Энергия активаций $\left(E_{A}\right)_{i}$ для $i$-ого закона в программе рассчитывается по уравнению Аррениуса для констант скоростей [5]:

$$
\ln k_{i k}=-\frac{\left(E_{A}\right)_{i}}{R} \cdot\left(\frac{1}{T_{k}}\right),
$$

где $R$ - универсальная газовая постоянная, $T_{k}$ - абсолютная температура в $K, k$ - порядковый номер исследуемой температуры, $k_{i k}-$ константа скорости для $i$-ого закона при $k$-ой температуре $T_{k}$. Энергия активации служит для определения зоны реакции при данной температуре: если $E_{A}<20$ кДж/моль, то зона реакции диффузионная, а если $E_{A}>50$ кДж/моль, то зона реакции кинетическая.

Средние погрешности аппроксимаций рассчитываются о формуле:

$$
\left(\varepsilon_{c p}\right)_{i k}=\frac{1}{n} \sqrt{\sum_{j=1}^{n} \frac{\left(\alpha_{j k}\left(t_{j}\right)-f_{i k}\left(t_{j}\right)\right)^{2}}{\alpha_{j k}{ }^{2}\left(t_{j}\right)}},
$$

где $\left(\varepsilon_{c p}\right)_{i k}$ - средняя погрешность аппроксимации $i$-ой функции при $k$-ой температуре $T_{k}, \alpha_{j k}\left(t_{j}\right)$ - экспериментальные значения степеней превращения вещества при температуре $T_{k}$ в моменты времени $t_{j}, f_{i k}\left(t_{j}\right)$ - расчётные значения пробной $i$-ой функции для наблюдаемого закона при температуре $T_{k}$ в момент времени $t_{j}$ и $n-$ количество отсчётов времени.

Расчёт погрешностей констант скоростей и порядков реакций производится в соответствии с [8]. Погрешности констант скоростей имеют интервальные оценки и рассчитываются как погрешности коэффициентов регрессии: 


$$
\begin{aligned}
& {\left[b_{1}^{*}-k_{p, \alpha} \cdot \frac{\sigma_{y}}{\sigma_{x}} ; b_{1}^{*}+k_{p, \alpha} \cdot \frac{\sigma_{y}}{\sigma_{x}}\right],} \\
& {\left[b_{0}^{*}-k_{p, \alpha} \cdot \frac{\sigma_{y}}{\sqrt{n}} ; b_{0}^{*}+k_{p, \alpha} \cdot \frac{\sigma_{y}}{\sqrt{n}}\right],}
\end{aligned}
$$

где $b_{i}^{*}$ - точечные оценки первого и второго коэффициентов регрессии, соответственно, $k_{p, \alpha}$ - критические значения распределения Стьюдента для уровня значимости $\alpha$ и для числа степеней свободы $p, \sigma_{y}$ - среднее квадратиче-

ское отклонение переменной $y$, вычисляемое по формуле $\sigma_{y}=\sqrt{\frac{\sum\left(y_{i}-\bar{y}\right)^{2}}{p}}$, где $y_{i}$ - значение выходной переменной $y$ в момент времени $t_{i}$, а $\bar{y}$ - её среднее значение, $\sigma_{x}=\sqrt{\sum\left(x_{i}-\bar{x}\right)^{2}}$.

Помимо расчётов кинетических параметров и их погрешностей в работе проводится проверка ряда статистических гипотез регрессионного и корреляционного анализов: гипотезы об адекватности регрессионной модели по критерию Фишера-Снедекора, о значимости коэффициентов регрессии по t-критерию Стьюдента и о точности предсказываемого отклика с использованием коэффициента корреляции и показателя согласованности Стьюдента [9].

Для проверки гипотезы об адекватности регрессионной модели по Снедекору-Фишеру сравнивается остаточная дисперсия, вычисляемая по

формуле $\sigma_{R}^{2}=\frac{\sum\left(y_{i}-y_{i}^{*}\right)^{2}}{p}$, где $y_{i}^{*}-$ значение выходной переменной, рассчитанное по уравнению регрессии; с дисперсией воспроизводимости $\sigma_{y}^{2}$.

Для сравнения рассеивания экспериментальных точек относительно линии регрессии с рассеиванием относительно математического ожидания используется F-отношение Снедекора-Фишера, которое, если $\sigma_{R}^{2}>\sigma_{y}^{2}$, вычисляется по формуле:

$$
f^{*}\left(k_{1}, k_{2}\right)=\frac{\sigma_{R}^{2}}{\sigma_{y}^{2}},
$$

где $k_{1}$ - число степеней свободы для остаточной дисперсии, $k_{2}-$ число степеней свободы для дисперсии воспроизводимости. Гипотеза об адекватности не отклоняется, если

$$
f^{*}\left(k_{1}, k_{2}\right)<f_{\alpha}\left(k_{1}, k_{2}\right)
$$

где $f_{a}\left(k_{1}, k_{2}\right)$ - значение в таблице F-распределения Фишера для уровня значимости $\alpha$ с числами степеней свободы $k_{1}$ и $k_{2}$, соответственно. Если $\sigma_{R}^{2}<\sigma_{y}^{2}$, то в формуле (6) в числителе должна быть $\sigma_{y}^{2}$ и число степеней сво- 
боды $k_{1}$ должно представлять собой число степеней свободы для $\sigma_{y}^{2}$, a $k_{2}-$ для $\sigma_{R}^{2}$.

В качестве нулевой гипотезы принимаем гипотезу $\mathrm{H}_{0}$ о том, что коэффициенты регрессии равны нулю при альтернативной гипотезе $\mathrm{H}_{1}$ о том, что коэффициенты не равны нулю. Проверка осуществляется с использованием t-критерия Стьюдента. Вычисляется статистика $t^{*}$ по формуле:

$$
t^{*}=\frac{b_{i}^{*}}{\sigma^{*}\left[b_{i}^{*}\right]},
$$

где $b_{i}^{*}$ - точечная оценка $i$-го коэффициента линейной регрессии, $\sigma^{*}\left[b_{i}^{*}\right]-$ точечная оценка среднеквадратического отклонения для $i$-го коэффициента линейной регрессии.

Расчётное значение статистики $t^{*}$ сравнивается с табличным значением $t_{\alpha}$ с заданным уровнем значимости $\alpha$. Если

$$
t^{*}<t
$$

то нулевая гипотеза не отклоняется.

Практическая значимость полученной регрессионной модели проверяется с использованием коэффициента корреляции

$$
r_{x y}=\frac{\sum_{i=1}^{n} x_{i} y_{i}-\frac{1}{n} \sum_{i=1}^{n} x_{i} \sum_{i=1}^{n} y_{i}}{\sqrt{\sum_{i=1}^{n} x_{i}{ }^{2}-\frac{1}{n}\left(\sum_{i=1}^{n} x_{i}\right)^{2}} \sqrt{\sum_{i=1}^{n} y_{i}{ }^{2}-\frac{1}{n}\left(\sum_{i=1}^{n} y_{i}\right)^{2}}}
$$

и показателя согласованности Стьюдента

$$
t_{r}=r_{x y} \sqrt{\frac{N-2}{1-r_{x y}^{2}}},
$$

где $x_{i}$ и $y_{i}$ - координаты экспериментальных точек.

Если коэффициент корреляции больше 0,7, то это указывает на тесную связь между входными и выходными переменными. Аналогично указывает на тесную связь и математическое неравенство

$$
t_{r}>t_{\alpha} \text {. }
$$

На основании изложенной выше теории первоначально создавался алгоритм, реализовывавшийся на основе Microsoft Access, и, впоследствии, создавалась программа Кинетика на языке Visual Basic, которая позволяла, используя методы регрессионного и корреляционного анализа, рассчитывать кинетические характеристики исследуемых реакций: константы скоростей, порядки реакций, энергии активации, зоны и механизмы реакций.

На рис. 1 в качестве примера показана вкладка Зона реакиии программы Кинетика с результатами расчётов по кинетике реакции фторирования нефелинового концентрата гидродифторидом аммония. Из рисунка видно, что 


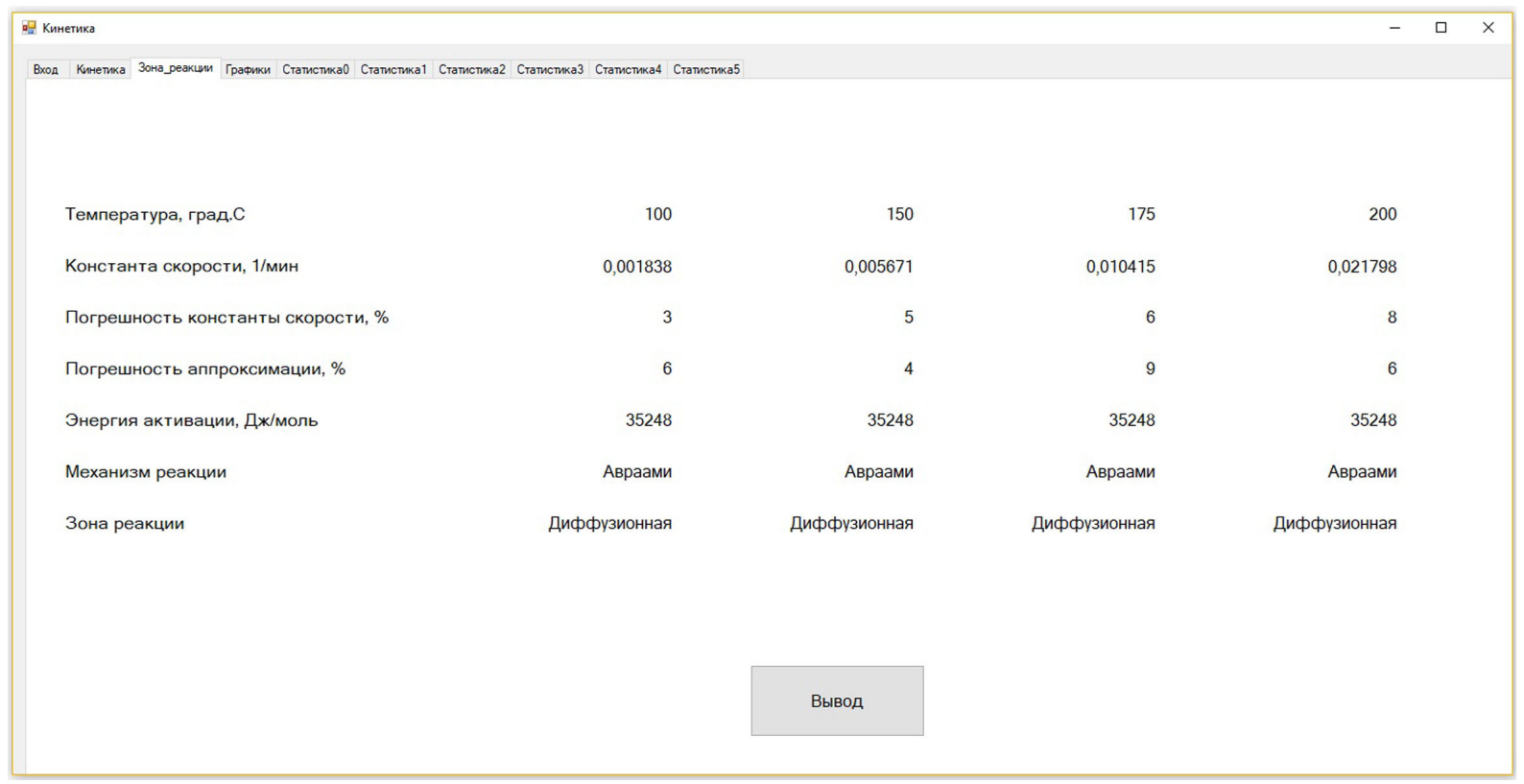

Рис. 1. Вкладка Зона реакции программы Кинетика с результатами расчёта для реакиии фторирования нефелинового кониентрата гидродифторидом аммония.

данная твёрдофазная реакция при всех температурах лучше всего описывается уравнением Авраами (даёт наименьшую погрешность аппроксимации). Поскольку энергия активации равна 35.2 кДж/моль, что больше 20кДж/ моль, но меньше 50кДж/моль, то зона реакции переходная. Константы скорости возрастают от 0.001838 мин $^{-1}$ до 0.021798 мин $^{-1}$.

Порядки реакции для Авраами принимают значения $0.71 ; 0.9 ; 0.94 ; 1.1$ (рис. 2). Поскольку порядки реакций дробные, приравняем их ближайшему целому числу. В данном случае это 1. Уравнение Авраами при порядке, равном 1, очевидно, переходит в экспоненциальный закон.

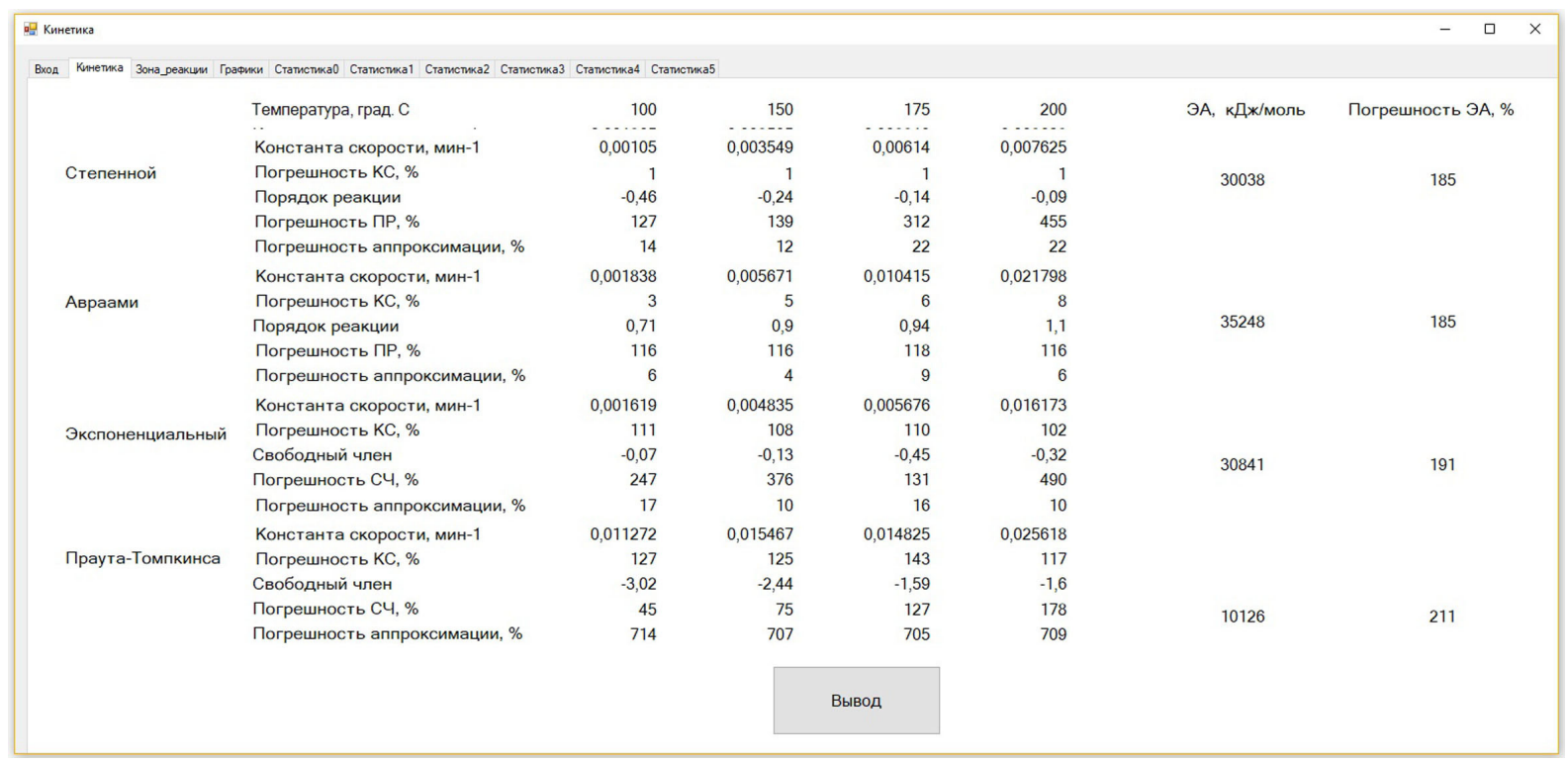

Рис. 2. Вкладка Кинетика программы Кинетика с результатами расчёта для реакции фторирования нефелинового концентрата гидродифторидом аммония. 
Результаты проверки, осуществляемой в программе, отражены в табл. 2. Проверка статистических гипотез в программе осуществляется для всех законов: её результаты размещены на вкладках СтатистикаХ. Мы рассмотрим, два из них: Авраами и экспоненциальный, поскольку они дают наилучшее приближение при всех температурах (см. табл. 2).

Таблица 2. Статистическая проверка гипотез об адекватности моделей регрессии и о значимости коэффициентов регрессий по Снедекору-Фишеру и Стьюденту, соответственно.

\begin{tabular}{|l|c|c|c|c|c|c|c|c|}
\hline \multicolumn{1}{|c|}{ Закон } & \multicolumn{4}{c|}{ Авраами } & \multicolumn{3}{c|}{ Экспоненциальный } \\
\hline \multicolumn{1}{|c|}{${\text { Температура, }{ }^{\circ} \mathrm{C}}^{10}$} & 100 & 150 & 175 & 200 & 100 & 150 & 175 & 200 \\
\hline $\begin{array}{l}\text { Средняя погрешность аппрок- } \\
\text { симации }\end{array}$ & 6 & 4 & 9 & 6 & 17 & 10 & 16 & 10 \\
\hline Коэффициент Фишера & 5.05 & 5.05 & 5.05 & 5.05 & 4.28 & 4.28 & 4.28 & 4.28 \\
\hline Статистика Фишера & 38.59 & 68.76 & 20.65 & 51.37 & 5.38 & 6.69 & 5.73 & 21.36 \\
\hline Коэффициент Стьюдента & 2.57 & 2.57 & 2.57 & 2.57 & 2.45 & 2.45 & 2.45 & 2.45 \\
\hline Статистика для своб. члена & 1.23 & 0.6 & 1.35 & 2.02 & & & & \\
\hline Статистика для угл. коэф. & 2.21 & 2.22 & 2.18 & 2.21 & 2.21 & 2.26 & 2.23 & 2.39 \\
\hline Коэффициент корреляции & 0.99 & 0.99 & 0.98 & 0.99 & 0.9 & 0.92 & 0.91 & 0.98 \\
\hline $\begin{array}{l}\text { Показатель согласованности } \\
\text { Стьюдента }\end{array}$ & 13.71 & 18.41 & 9.91 & 15.87 & 4.68 & 5.33 & 4.86 & 10.09 \\
\hline
\end{tabular}

Статистики Фишера для обоих законов при всех температурах больше, чем коэффициенты Фишера, поэтому гипотеза об адекватности регрессионных моделей в обоих случаях отвергается, правда, статистики в случае экспоненциального закона ближе к коэффициентам Фишера.

Коэффициенты корреляции между входными и выходными данными для обоих законов больше 0.7. Показатели согласованности также превосходят критические значения распределения Стьюдента, следовательно, гипотеза об отсутствии тесной связи между входными и выходными данными отвергается, и функции отклика для обоих законов могут использоваться для предсказания результата.

Проверка гипотезы о значимости коэффициентов регрессии при помощи t-критерия Стьюдента показывает, что как статистики для свободных членов, так и для угловых коэффициентов Авраами меньше соответствующих коэффициентов Стьюдента, а, следовательно, являются статистически незначимыми. Однако, заметим, что статистики для углового коэффициента близки к коэффициентам Стьюдента, a, следовательно, мы можем допустить их значимость, т.е. считать, что они не равны нулю. Вспомнив, что угловые коэффициенты в случае Авраами равны порядкам реакции, будем считать порядки реакции равными 1. В случае с экспоненциальным законом статистики для углового коэффициента также близки к коэффициентам Стьюдента, будем считать угловые коэффициенты значимыми также и для экспоненциального закона. Поскольку угловые коэффициенты для экспоненциально- 
го закона равны константам скоростей, будем иметь константы скоростей для нашей реакции.

Однако, имея ввиду, что лучшую аппроксимацию дает уравнение Авраами, возможно, следует взять средние значения констант скоростей между двумя законами с погрешностями, равными суммам погрешностей. По этим усреднённым константам скоростей рассчитаем энергии активации. Поместим эти данные в табл. 3.

Заметим, что энергия активации реакции получается равной 33.6 кДж/ моль, т.е. реакция проходит в переходной зоне, как и следовало ожидать. Следовательно, химическая реакция фторирования нефелинов гидродифторидом аммония является реакцией первого порядка, следовательно, сопротивление реакции и диффузии приблизительно одинаковы и оказывают одинаковое влияние на ход реакции. Константы скоростей реакции при всех температурах приведены в табл. 3.

Реакция взаимодействия двух веществ, нефелина и гидродифторида аммония, имеет первый порядок. Такое может быть, когда реакция протекает при избытке одного из веществ. Возможно также протекание реакции в несколько стадий. В первую стадию происходит быстрая реакция взаимодействия этих двух реагентов с образованием активационного комплекса. Распад же комплекса протекает медленно, возможно, не за одну стадию. Ход реакции, по-видимому, определяется стадией, в которой происходит разложение активационного комплекса.

Таблица 3. Кинетические характеристики реакции фторирования нефелинов гидродифторидом аммония.

\begin{tabular}{|c|c|c|c|c|c|}
\hline & Температура, ${ }^{\circ} \mathrm{C}$ & 100 & 150 & 175 & 200 \\
\hline \multirow{4}{*}{ Авраами } & Константы & $0.001838 \pm$ & $0.005671 \pm$ & $0.010415 \pm$ & $0.021798 \pm$ \\
\hline & скоростей, мин ${ }^{-1}$ & 0.0001 & 0.0003 & 0.0006 & 0.002 \\
\hline & Порядки реакций & 0.71 & 0.9 & 0.94 & 1.1 \\
\hline & $\begin{array}{c}\text { Энергия активации, } \\
\text { кДж/моль }\end{array}$ & \multicolumn{4}{|c|}{35.2} \\
\hline \multirow{4}{*}{ Экспоненциальный } & Константы & $0.001619 \pm$ & $0.004835 \pm$ & $0.005676 \pm$ & $0.016173 \pm$ \\
\hline & скоростей, мин ${ }^{-1}$ & 0.002 & 0.005 & 0.006 & 0.02 \\
\hline & Порядки реакций & \multicolumn{4}{|c|}{1} \\
\hline & $\begin{array}{c}\text { Энергия активации, } \\
\text { кДж/моль }\end{array}$ & \multicolumn{4}{|c|}{30.8} \\
\hline \multirow{4}{*}{$\begin{array}{c}\text { Фторирование } \\
\text { нефелинов ГДФА }\end{array}$} & Константы & $0.0017 \pm$ & $0.0053 \pm$ & $0.0080 \pm$ & $0.019 \pm$ \\
\hline & скоростей, мин ${ }^{-1}$ & 0.0021 & 0.0053 & 0.0066 & 0.022 \\
\hline & Порядки реакций & \multicolumn{4}{|c|}{1} \\
\hline & $\begin{array}{c}\text { Энергия активации, } \\
\text { кДж/моль }\end{array}$ & \multicolumn{4}{|c|}{33.6} \\
\hline
\end{tabular}




\section{Список литературы}

1. Пушкин А.А. Римкевич В.С. Автоматизация расчётов изменений термодинамических потенциалов в ходе химических реакций // Межд. науч.исслед. журнал. 2015. № 6 (37). Ч. 1. С. 108-119.

2. Пушкин А.А., Римкевич В.С. Программа для расчёта кинетики гетерофазных реакций на языке Visual Basic Community 2015 // Фундаментальные исследования. 2017. № 10. Ч. 3. С. 518-523.

3. Бочкарев В.В. Теория химико-технологических процессов органического синтеза. Гетерофазные и гетерогенно-каталитические реакции. Уч. пос. Томск: Изд-во ТПУ. 2005. 118 с.

4. Евдокимов А.Н. Теория химико-технологических процессов органического синтеза. Ч. 1. Гетерофазные реакции. Уч. пос. С-Пб. 2011. 64 с.

5. Еремин Е.Н. Основы химической кинетики: Уч. пос. М.: Высш. шк. $1976.375 \mathrm{c}$.

6. Стромберг А.Г., Семченко Д.П. Физическая химия. М.: Высшая школа. 1999. $528 \mathrm{c}$.

7. Пушкин А.А., Римкевич В.С. Установление зон гетерофазных реакций // Международный научно-исследовательский журнал. 2017. № 03 (57). Ч. 3. C. 35-38.

8. Колемаев В.А., Староверов С.В., Турундаевский В.Б. Теория вероятностей и математическая статистика. Уч. пос. М.: Высшая школа. 1991. 400 с. 9. Балдин К.В., Башлыков В.Н., Рукосуев А.В. Теория вероятностей и математическая статистика. Учебник. М.: Изд-во торг. корпорация «Дашков и $\mathrm{K}^{\circ} \gg .2014 .473 \mathrm{c.}$ 